\title{
Enhanced Depth imaging optical coherence tomography of the choroid in eyes with high myopia and normal control group of kashmiri population
}

\author{
Authors \\ Dr Nazia Anjum, Prof. Junaid S. Wani, Prof. Afroz A. Khan, Dr Aalia Rasool \\ Dr Birjees Hakak
}

\begin{abstract}
Background: The purpose of this study was to examine the choroidal thickness $(C T)$ of patients with high myopia using enhanced depth imaging optical coherence tomography(EDI-OCT) and compare with healthy subjects.

Methods: A case-control study of 107 eyes (57patients) with high myopia and 60 eyes (30 patients) of emmetropes. Choroidal imaging was performed using enhanced depth imaging spectral domain optical coherence tomography. CT was measured at, subfovea, $1 \mathrm{~mm}$ and $3 \mathrm{~mm}$ nasal and temporal to fovea.

Results: Statistical analysis was performed to evaluate CT at different locations and to correlate CT with axial length $(A L)$ and spherical equivalent(SE).Mean SE was -12.07+-3.184D in eyes with high myopia and $25.66+-8.39 D$ in normal control group. Mean AL was $26.68+-1.577 \mathrm{~mm}$ in eyes with high myopia and $22.88+-0.56 \mathrm{~mm}$ in normal control group. The CT at the subfovea was significantly thinner (mean+standard error: 192.79+-51.61 $\mu \mathrm{m})$ for high myopic eyes compared to emmetropes $(305.30+-60.11 \mu \mathrm{m}$, p $<0.001)$.Likewise, CT in high myopic group was significantly thinner than emmetropic control group at all locations ( $p$ for trend $<0.001$ for all locations). Choroid was thinnest at nasal $3 \mathrm{~mm}$ location in both the myopic $(136.46+-50.33 \mu \mathrm{m})$ and emmetropic $(289.02+-60.15 \mu \mathrm{m})$ groups. Choroid was thickest subfoveally in both the myopic $(192.79+-51.61 \mu \mathrm{m})$ and emmetropic $(305.30+-60.11 \mu \mathrm{m})$ groups. CT was negatively correlated with $S E$ and $A L$.

Conclusions: High myopic eyes have significantly thinner choroid compared to emmetropic eyes. Axial length and spherical equivalent are significant predictors of choroidal thickness.

Keywords: Myopia, OCT, Choroidal thickness.
\end{abstract}

\section{Introduction}

Myopia or short sightedness is that form of refractive error wherein parallel rays of light come to a focus in front of the sentient layer of the retina when the eye is atrest. ${ }^{1}$

High myopia(defined as myopia of $-6 \mathrm{D}$ or more) is one of the main causes of visual impairment worldwide. ${ }^{2}$ About $1 \%$ of the population suffers from this disease. ${ }^{3}$
High myopia is always accompanied by pathological structural changes such as axial elongation, posterior staphyloma, lacquer crack formation, thinning of the retina and choroid, and choroidal neovascularization. ${ }^{4,5,6}$ One of the main cause of the ocular complications is excessive axial elongation of the eye ball.

The choroid, primarily a vascular structure, has several important functions in the eye including 
delivery of blood and nutrients to the outer retina, thermoregulation of the retina, and secretion of growth factors. ${ }^{7}$ The ciliary muscle transmits tension to the choroid during accommodation, and this has been proposed as a possible mechanical stimulus contributing to myopia development. Since ciliary body thickness is greater in myopic compared to non-myopic children and increases with increasing axial length in adults, it has been hypothesized that a thickened, poorly contracting ciliary muscle could lead to accommodative dysfunction and a lag of accommodation (i.e. hyperopic retinal defocus during near work), which subsequently stimulates axial elongation and choroidal thinning.

Choroidal thickness (CT) may be an important parameter in studying the pathogenesis of high myopia. ${ }^{8,9,10,11,12} \quad$ Spectral-domain optical coherence tomography(SD-OCT) is a powerful modality for investigating the retinal/choroidal structure of highly myopic eyes. ${ }^{13}$ The aim of our study was to measure CT in high myopic eyes and in emmetropes, to compare CT in myopic eyes with normal control eyes, and to correlate CT with axial length(AL) and spherical equivalent(SE).

\section{Material and Methods}

It was a hospital based observational, prospective, comparative study. The macular choroidal thickness of high myopic eyes attending the OPD of Postgraduate Department of Ophthalmology, Government Medical College, Srinagar, and normal eyes as controls were measured using enhanced depth imaging-optical coherence tomography (EDI-OCT).The study was conducted from September 2017 to September 2019.Study was approved by Ethical review committee.

In the high myopia group, the inclusion criteria were as follows: (1) high myopia was defined as SE >-6 diopters (D); (2) all eyes with clear ocular media;(3) a clear image was obtained to enable precise measurements of choroidal thickness.

Patients were excluded from this study if they presented with any retinal abnormalities other than high myopia such as diabetic retinopathy, retinal vascular abnormalities, age related macular degeneration, laser or anti-VEGF therapy, patients with history of intraocular surgery, refractive surgery, glaucoma, amblyopia and media opacity. All the patients underwent refractive error examination without pupil dilatation using autorefraction (VISUREF-100, make CARL ZEISS) which was confirmed by manifest refraction. Spherical equivalent was calculated as the sum of spherical power and half of the cylinder. All eyes underwent a thorough ophthalmic evaluation, including intraocular pressure measurement (IOP/NCT - make AT 555), slit lamp biomicroscopy, dilated fundus examination (indirect ophthalmoscopy), and axial length measurement (LENSTAR LS 900).

\section{EDI-OCT Examination}

All patients were examined with SD-OCT machine with an EDI mode (Cirrus HD-OCT, 5000). Images of the choroidal thickness were obtained by positioning the device close enough to the eye to acquire an enhanced signal of the choroidal layer. ${ }^{13}$ If an OCT instrument is positioned closer to the eye, an inverted mirror image is obtained. This inverted mirror image has more information from the deep choroid than the normal non-inverted image. The most recent version of Spectralis software incorporates EDI into the scanning protocols. With the EDI mode, the software automatically inverts the image. Choroidal thickness was measured as the distance between the hyper reflective retinal pigment epithelium (RPE) layer and the inner scleral border. Choroidal thickness was measured at the sub fovea, $1 \mathrm{~mm}$ and $3 \mathrm{~mm}$, nasal and temporal to fovea and was measured manually using the caliper tool. To avoid possible diurnal variation in $\mathrm{CT}$, all EDI-OCT examinations were performed between 10 am to $2 \mathrm{pm}$.

\section{Statistical analysis}

Descriptive statistics including mean, standard deviation, minimum and maximum for continuous data and frequency and percentage for categorical variable were used. Student's independent t-test was employed for comparing continuous 
variables. A P-value of less than 0.05 was considered statistically significant. All P-values were two tailed.

\section{Results}

A total of 107 eyes of 57 highly myopic patients and 60 eyes of 30 normal participants were included in the study. In 5 patients only leye was included in view of unilateral high myopia and 2 eyes were excluded because the border between the choroid and the sclera could not be visualized, although the optical media was clear. Therefore data from 107 eyes of 57 high myopic patients and
60 eyes of 30 normal participants with high quality OCT images were available and included in the analysis. The mean age was $28.54+9.44$ years in high myopia patients and $25.66+8.3$ in control subjects. The mean SE was $-12.07+3.18$ $\mathrm{D}$ in high myopia group and $-0.03+0.195 \mathrm{D}$ for the control group. The mean AL was $26.68+1.57 \mathrm{~mm}$ in high myopia group and $22.88+0.56 \mathrm{~mm}$ in the control group. The demographic and baseline characteristics are summarized in table 1.There was a significant between the two groups in $\mathrm{SE}(\mathrm{P}<0.001)$ and $\mathrm{AL}$ $(\mathrm{P}<0.001)$.

Table 1: Demographic and baseline characteristics of study subjects

\begin{tabular}{|l|c|c|c|}
\hline & High myopia & Normal control & P value \\
\hline NO. of patients (No. of eyes) & $57(107)$ & $30(60)$ & \\
\hline Mean age (SD),Y & $28.54(9.44)$ & $25.66(8.39)$ & \\
\hline Spherical equivalent (SD),D & $-12.07(3.184)$ & $-0.03(0.195)$ & $<0.001$ \\
\hline Axial length(SD),mm & $26.68(1.577)$ & $22.88(0.559)$ & $<0.001$ \\
\hline
\end{tabular}

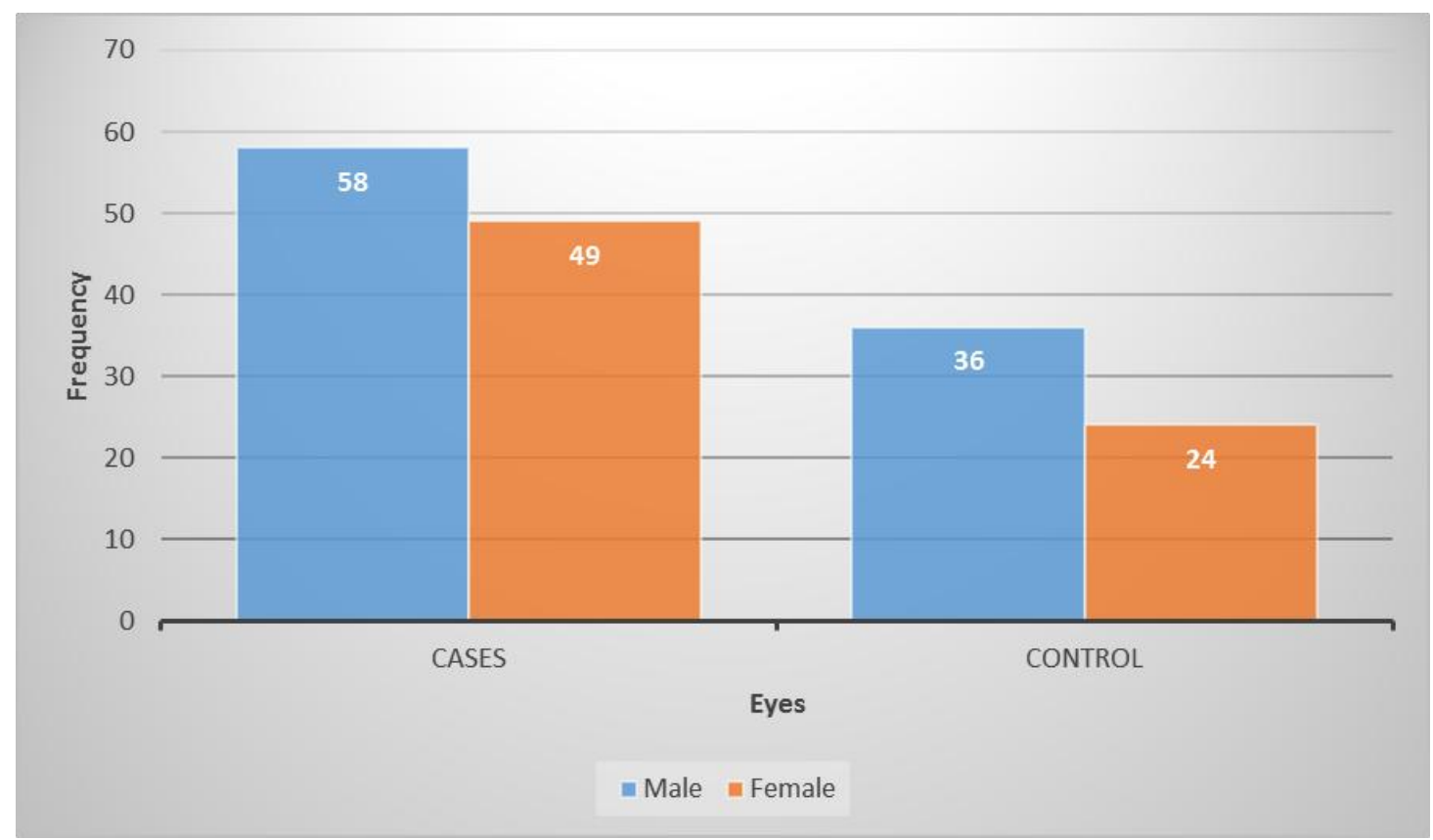

Figure-1A: Gender wise distribution of eyes in cases and control 


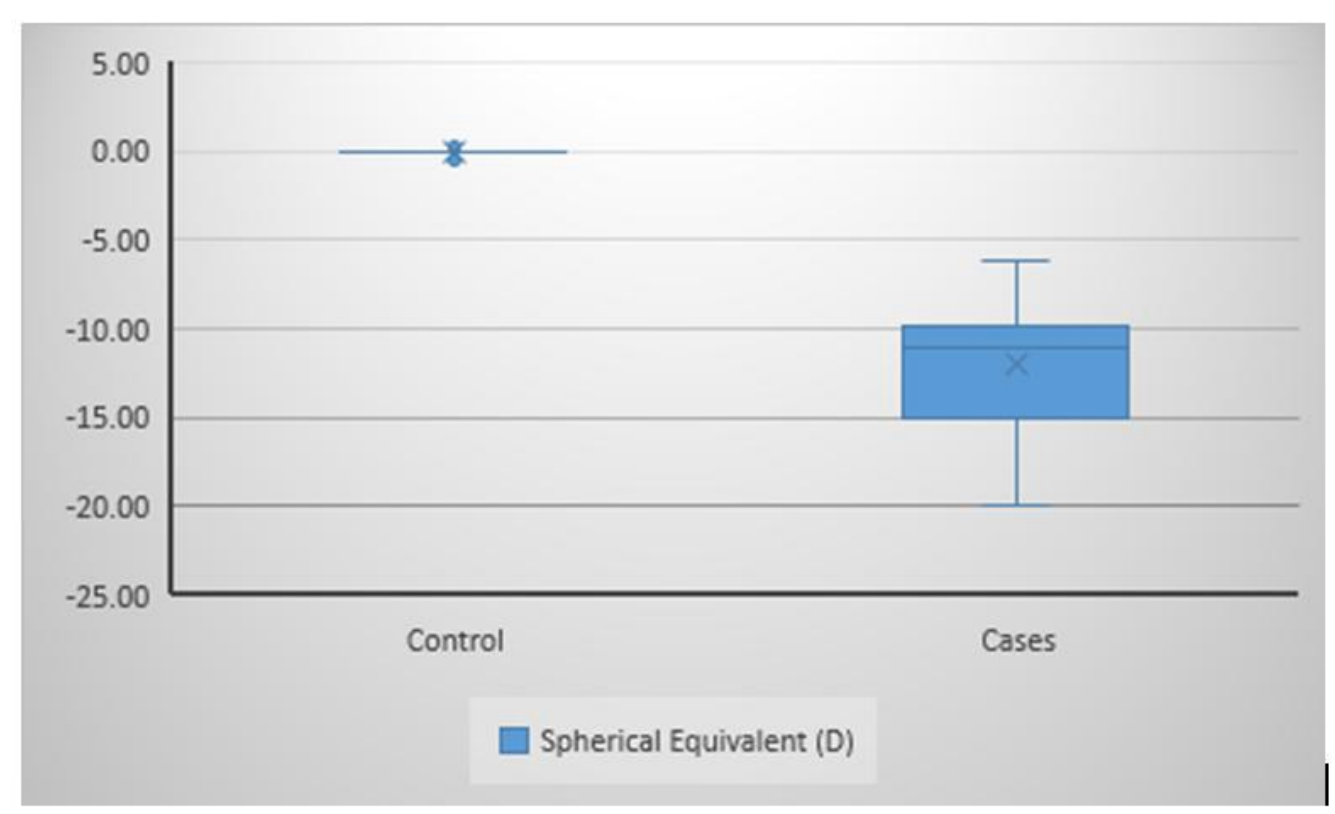

Figure 1B.Comparison of Spherical equivalent in cases and control

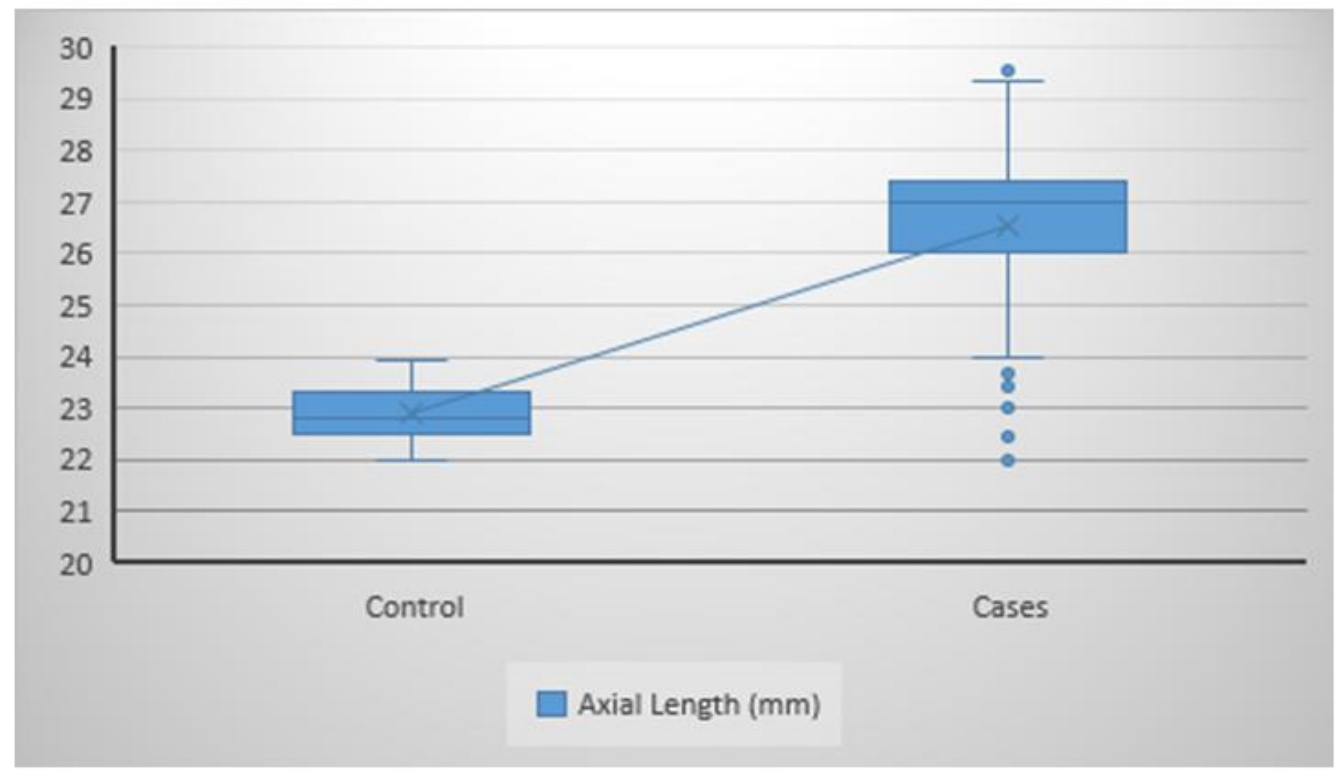

Figure 1C. Comparison of Axial length in cases and control

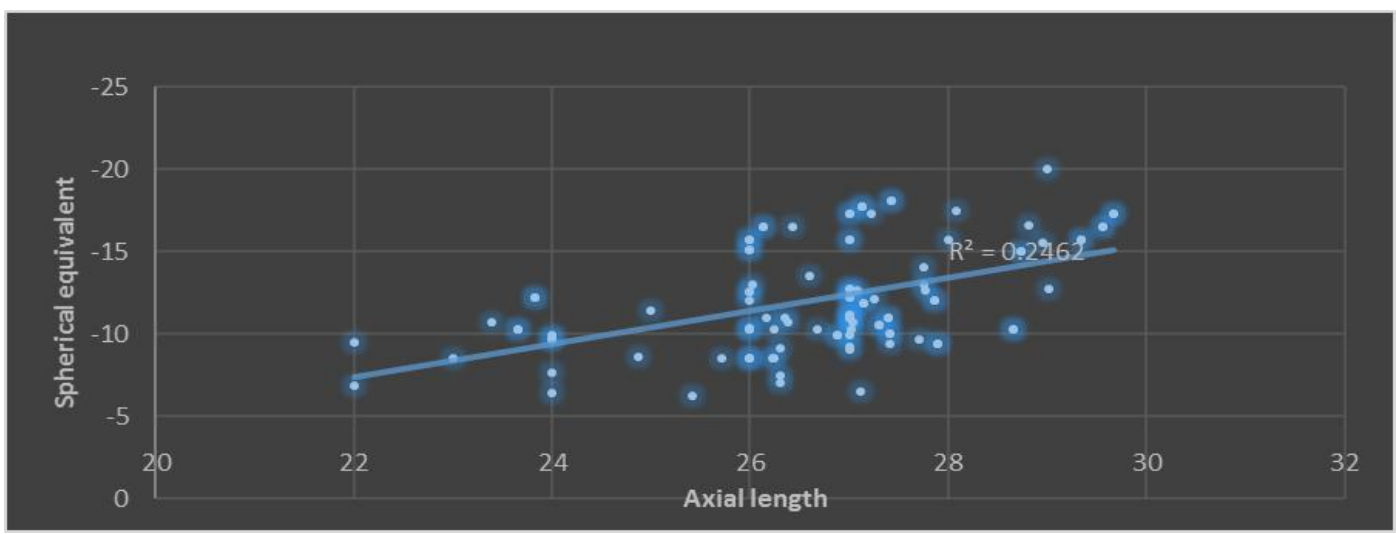

Figure 1D Scatter plot showing comparison of Spherical equivalent \& Axial length in cases. 
Table 2: Choroidal thickness at different locations in myopic and control group

\begin{tabular}{|l|c|c|c|c|c|c|}
\hline & & $\begin{array}{c}\text { Subfoveal } \\
(\mathbf{S F})\end{array}$ & $\begin{array}{c}\text { Nasal } \\
\mathbf{1 m m}\end{array}$ & $\begin{array}{c}\text { Nasal } \\
\mathbf{3 m m}\end{array}$ & $\begin{array}{c}\text { Temporal } \\
\mathbf{1 m m}\end{array}$ & $\begin{array}{c}\text { Temporal } \\
\mathbf{3 m m}\end{array}$ \\
\hline \multirow{4}{*}{ Cases } & Min & 65 & 48 & 46 & 64 & 60 \\
\cline { 2 - 7 } & Max & 296 & 279 & 278 & 292 & 282 \\
\cline { 2 - 7 } & Mean & 192.79 & 160.98 & 136.46 & 167.19 & 161.86 \\
\cline { 2 - 7 } & SD & 51.61 & 53.03 & 50.33 & 47.55 & 43.38 \\
\hline \multirow{4}{*}{ Controls } & Min & 200 & 185 & 180 & 195 & 191 \\
\cline { 2 - 7 } & Max & 391 & 380 & 378 & 388 & 387 \\
\cline { 2 - 7 } & Mean & 305.30 & 292.17 & 289.02 & 299.38 & 295.37 \\
\cline { 2 - 7 } & SD & 60.11 & 60.15 & 60.38 & 60.01 & 60.87 \\
\hline
\end{tabular}

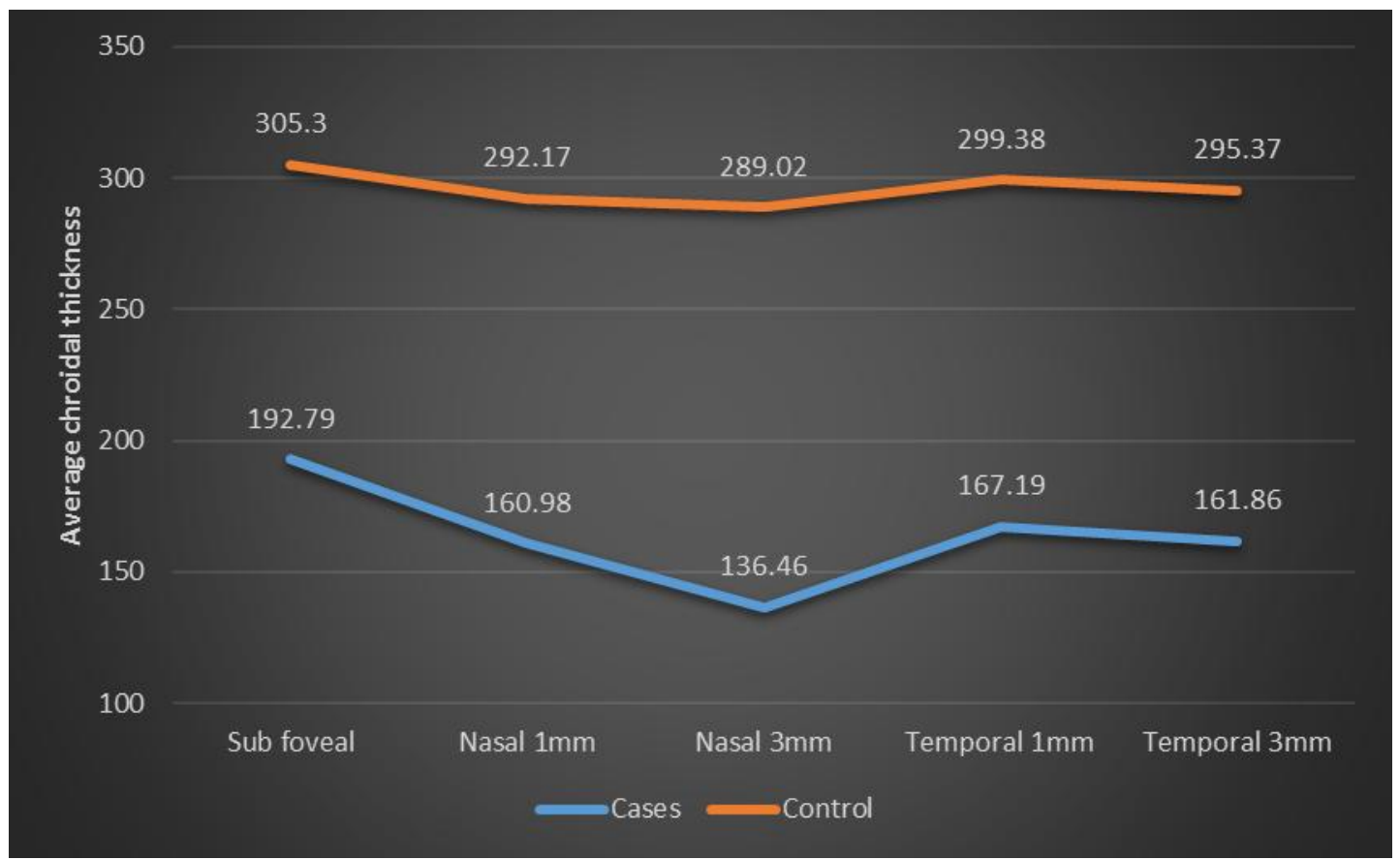

Figure 2A Graph showing mean $\mathrm{CT}$ at different location in cases and control

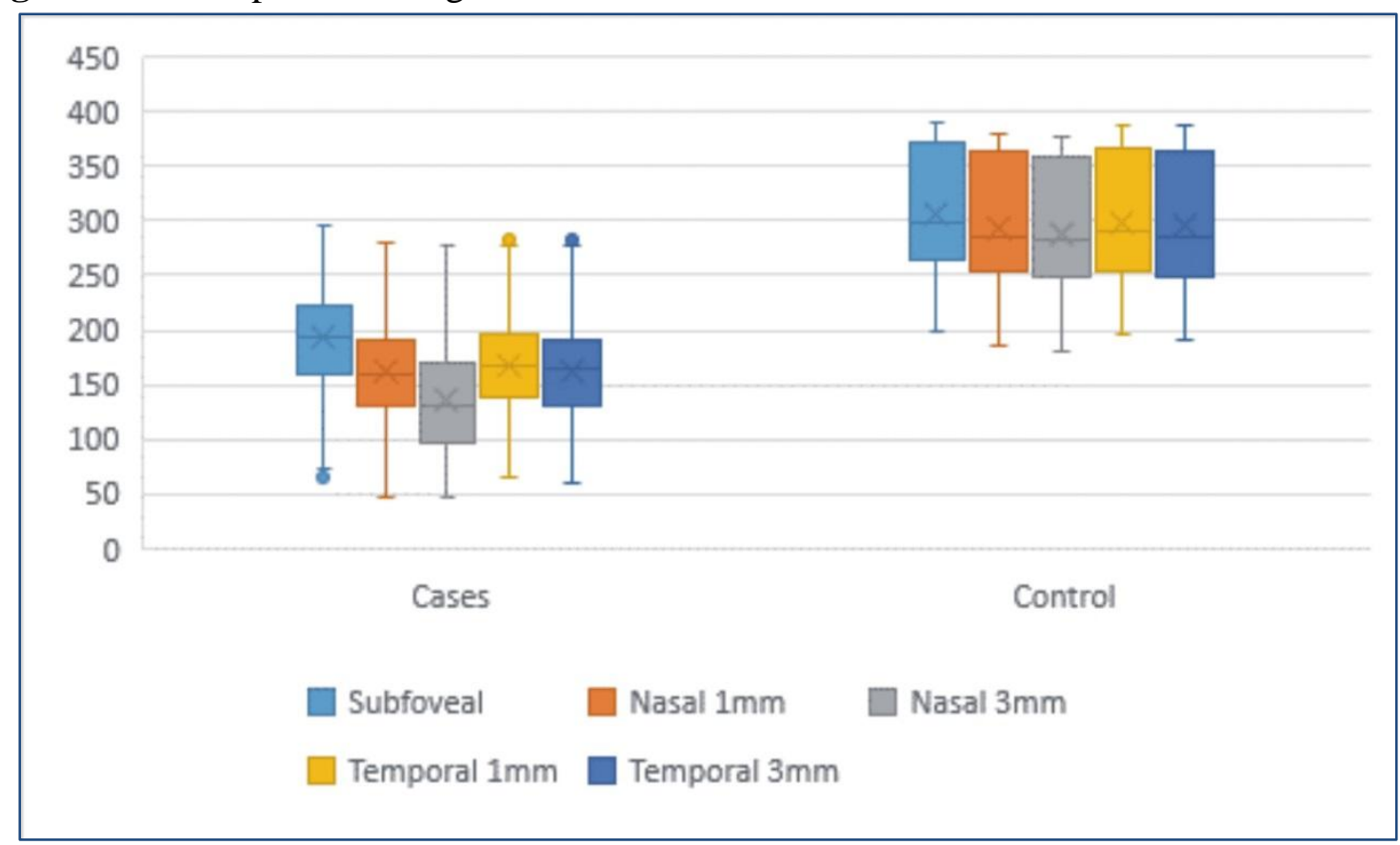

Figure 2B Whisker graph showing comparison of CT at different locations in cases and control 
CT varied significantly across the myopic patients and emmetropic control group at all locations ( $P$ for trend $<0.001$ for all locations, table 2).It was thinner in myopic eyes compared to control group. CT was thickest subfoveally (SF) and thinnest nasally in both high myopic eyes and in normal control eyes ( $p$ value, 0.001 by repeated measures ANOVA).However, in both the high myopia and emmetropic groups, choroid was thinnest at the nasal location being $136.46 \mu \mathrm{m}$ and $289.02 \mu \mathrm{m}$ in myopic and emmetropic group respectively (Fig. 1A,B). CT was found to have negative correlation with SE and AL.

\section{Discussion}

In our study, choroidal thickness was measured at the sub fovea, $1 \mathrm{~mm}$ and $3 \mathrm{~mm}$ nasal, $1 \mathrm{~mm}$ and $3 \mathrm{~mm}$ temporal to fovea in cases and control on HD OCT by EDI mode. The images were obtained with the best visualization of the border between the choroid and the sclera known as the choroid scleral interface (CSI), if neither image had a clearly identifiable CSI, additional images were taken to produce best possible view of the CSI.

Overall, choroidal thickness was significantly lower in eyes with high myopia compared to normal control eyes which is consistent with the findings of Wang S et al (2015) ${ }^{14}$, Gupta $\mathrm{P}$ et al $(2015)^{15}$, Fujiwara $\mathrm{T}$ et al (2009) ${ }^{16}$ and ElZawahry et al $(2017)^{17}$.

We found that the thickest regions were found in sub foveal area and thinnest regions were found in the nasal area both in eyes with high myopiaand inthe normal control eyes.

In the more and less myopic eyes, the choroid was thinnest in the nasal region and thickest in the foveal-temporal region (Vincent SJ et al., 2013) ${ }^{18}$, which is in general agreement with previous studies examining CT in healthy eyes. It has been suggested that this region of thinner choroid nasal to the fovea (i.e., between the fovea and optic nerve) is most susceptible to change during myopia development. Two possible reasons for the relative choroidal thinning nasally and inferiorly in normal eyes are the choroidal watershed and fetal choroidal fissure, which closes here at 7 weeks. ${ }^{19}$

Among the ocular factors studied axial length, and spherical equivalent were the significant predictors of choroidal thickness (CT). Wang S et al $(2015)^{14}$ in his study stated that in high myopic eyes, excessive elongation of the eyeballs can cause biomechanical stretching and thinning of choroid, retina and sclera. Thus, the thinner choroid is a long term correlate in high myopic eyes might be secondary in its effects to the longer axial length, but it is not an independent factor. Thinning of the sclera has been described in high myopia, with narrowing and dissociation of the collagen fiber bundles, a reduction in collagen fibre diameter, a reduction in extracellular matrix, and a loss of the normal organization of collagen fibres. The retina has been reported to undergo degenerative changes as well with thinning of macula. Histologic studies of highly myopic eyes have shown that the choroid is thin with a paucity of large choroidal vessels and loss of the choriocapillaries. Choroidal degeneration as a direct cause of increased axial length has been reported in an animal model in which decreased choriocapillaries density diameter was observed, as well as absence of endothelial fenestrations and narrowed lumen .These findings have led to the theory that axial elongation in high myopia results in stretching of the ocular coats, which has been supported by experimental models of induced myopia $^{16}$.

These findings suggest that choroidal thinning may play a role in the pathophysiological features of vision loss in high myopia, just as a relative ischemia of the outer retina, RPE, and the choroid itself may lead to pathological decompensation. It seems likely that after the supply offered by the choroid is reduced to very low levels, ophthalmoscopically evident changes, such as broad areas of depigmentation and what is termed myopic atrophy or degeneration, may become visible. 
The study has several strengths. Our study is done in kashmiri population first time, and EDI SDOCT images were enhanced using adaptive compensation (Wang et al.1994) to improve CSI visibility. Therefore, the CT measurement obtained in our study are likely more reliable and accurate. Limitations to this study are, CT measurements were obtained manually because currently there is no automated software to measure CT.Studies of choroidal circulation are needed to correlate CT with perfusion.

\section{Conclusion}

Choroid is thickest subfoveally and thinnest nasally in both cases and controls but thinning is more in cases at all areas.

Axial length and spherical equivalent are significant predictors of choroidal thickness and must be taken into account when interpreting the data on choroidal thickness.

\section{Bibliography}

1. Duke-Elder's Practice of Refraction, 10th Edition, P 53.

2. Klaver CC, Wolfs RC, Vingerling JR, Hofman A, de Jong PT. Age specific prevalence and causes of blindness and visual impairment in an older population: the Rotterdam Study. Arch Ophthalmol 1998, 116: 653-58.

3. Vongphanit J, Mitchell P, Wang JJ. Prevalence and progression of myopic retinopathy in an older population. Ophthalmology. 2002; 109: 704-11.

4. Lim MC, Hoh ST, Foster PJ, Lim TH, Chew SJ, Seah SK, et al. Use of optical coherence tomography to assess variations in macular retinal thickness in myopia. Invest Ophthalmol Vis Sci. 2005; 46: 9748.

5. Curtin BJ, Karlin DB. Axial length measurements and fundus changes of the myopic eye. Am J Ophthalmol. 1971; 71: 42-53.
6. Takano M, Kishi S. Fovealretinoschisis and retinal detachment in severely myopic eyes with posterior staphyloma. Am J Ophthalmol. 1999; 128: 472-6.

7. Nickla D, Wallman J. The multifunctional choroid. ProgRetin Eye Res 2010; 29(2): 144-168.

8. Hamzah F, Shinojima A, Mori R, Yuzawa M. Choroidal thickness measurement by enhanced depth imaging and swept-source optical coherence tomography in central serous chorioretinopathy. BMC Ophthalmol. 2014; 14: 145.

9. Zhou M, Wang W, Ding X, Huang W, Chen S, Laties AM, et al. Choroidal thickness in fellow eyes of patients with acute primary angle-closure measured by enhanced depth imaging spectraldomain optical coherence tomography. Invest Ophthalmol Vis Sci. 2013; 54: 1971-8.

10. Huang W, Wang W, Zhou M, Chen S, Gao $\mathrm{X}$, Fan Q, et al. Peripapillary choroidal thickness in healthy Chinese subjects. BMC Ophthalmol. 2013; 13: 23.

11. Zhou M, Wang W, Huang W, Gao X, Li Z, $\mathrm{Li} X$, et al. Is increased choroidal thickness association with primary angle closure? ActaOphthalmol. 2014; 92: e514-20.

12. Hirooka K, Tenkumo K, Fujiwara A, Baba T, Sato S, Shiraga F. Evaluation of peripapillary choroidal thickness in patients with normal-tension glaucoma. BMC Ophthalmol. 2012; 12: 29.

13. Margolis R, Spaide RF. A pilot study of enhanced depth imaging optical coherence tomography of the choroid in normal eyes. Am J Ophthalmol. 2009; 147(5): 811-815.

14. Wang S, Wang Y, Gao X, Qian N, Zhou Y. Choroidal thickness and high myopia: a cross-sectional study and meta-analysis. BMC Ophthalmology 2015; 15: 70.

15. Gupta P, Saw SM, Carol Y. Cheung, Michael J. A. Girard, Jean Martial Mari, Mayuri Bhargava et al. Choroidal thickness and high myopia: a case-control 
study of young Chinese men in Singapore. Acta Ophthalmologica 2014: 1-8.

16. Fujiwara T, Imamura $\mathrm{Y}$, Margolis R, Slakter JS \&Spaide RF. Enhanced depth imaging optical coherence tomography of the choroid in highly myopic eyes. Am J Ophthalmol 2009; 148: 445- 50.

17. El-Zawahry WM. Subfoveal choroidal thickness versus foveal thickness in patients Vincent SJ, Collins MJ, Read SA, Carney LG. Retinal and choroidal thickness in myopic anisometropia. Invest Ophthalmol Vis Sci. 2013 Apr; 54(4): 2445-56.

18. Vincent SJ, Collins MJ, Read SA, Carney LG. Retinal and choroidal thickness in myopic anisometropia. Invest Ophthalmol Vis Sci. 2013 Apr; 54(4): 2445-56.

19. Sadler TW (2006): Langman's medical embryology. Baltimore, MD: Lippincott Williams and Wilkins. 